\title{
MODELLING OF DIAGONAL COMPRESSION MASONRY WALLETS BY FINITE ELEMENTS WITH EMBEDDED DISCONTINUITIES
}

\author{
Gelacio Juárez-Luna ${ }^{1}$ and Arturo Tena-Colunga ${ }^{2}$ \\ ${ }^{1,2}$ Departamento de Materiales, Universidad Autónoma Metropolitana \\ San Pablo 180, Azcapotzalco, México D.F. 02200, Mexico \\ e-mail: ${ }^{1}$ gjl@azc.uam.mx ${ }^{2}$ atc@azc.uam.mx
}

Keywords: Wallets, Combined Masonry, Embedded Discontinuities, Cracking, Damage.

\begin{abstract}
This paper investigates the behaviour of small square masonry subassembly, wallets, subject to axial compression, involving their crack pattern and the shear stress-shear strain curves. Masonry and mortar were discretized with quadrilateral finite elements with embedded discontinuities, which have three degrees of freedom per node. The constitutive behaviours of blocks, bricks and mortar consider the softening deformation after reaching a failure surface. The numerical modelling of experimental tests for combined masonry wallets were performed in the Finite Element Analysis Program (FEAP). The computed shear stressshear strain curves with finite elements with embedded discontinuities showed a fairly good agreement in terms of the initial stiffness, strength, ultimate load and dissipative behaviour; also, the computed cracking patter is congruent with the experimental one.
\end{abstract}




\section{INTRODUCTION}

The modelling of masonry structures is a demanding task in structural engineering. This laborious work is attributed to the geometry and to the constitutive behaviour of bricks and mortar materials, which include anisotropy and nonlinearity. The different numerical procedures could be summarized in three levels of refinement [2][3]: 1) macro-modelling, bricks, mortar and the brick-mortar interface are smeared out in a homogeneous continuum; 2) simplified micro-modelling, bricks are represented as fictitious expanded bricks by continuum elements with the same size as the original bricks dimensions plus the real joint thickness. The mortar joint is also discretized with interfaces of zero thickness; and 3) detailed micromodelling, units and mortar in the joints are discretized by continuum elements whereas the brick-mortar interface is represented by discontinuous elements.

This papers uses a kind of the detailed micro-modelling, where perfect bond in the brickmortar interface was assumed. Small square masonry wallets, subject to axial compression, were modelled. Bricks, block and mortar were discretized with hexahedral finite elements with embedded discontinuities (FEED). The constitutive behaviour of blocks, bricks and mortar considers the softening deformation after reaching a discrete failure surface. The computed shear stress-shear strain curves with FEED are stiffer than the experimental curves, a fact attributed that the sliding in the brick-mortar interface was neglected.

\section{EMBEDDED DISCONTINUITY MODEL}

\subsection{Variational formulation}

The FEED are formulated from an energy functional which has the displacement, $\mathbf{u}$, and the displacement jump [|u|] as independent variable ([1], [5], [6], [12]). This functional is given by:

$$
\Pi(\mathbf{u},[|\mathbf{u}|])=\int_{\Omega \backslash S}\left[\Psi\left(\overline{\boldsymbol{\varepsilon}}^{u}\right)-\mathbf{b} \cdot \mathbf{u}\right] d \Omega-\int_{\Gamma_{\sigma}} \mathbf{t}^{*} \cdot \mathbf{u} d \Gamma+\int_{\Gamma_{S}} \phi_{S}([|\mathbf{u}|]) d \Gamma
$$

where the free energy density, $\Psi\left(\bar{\varepsilon}^{u}\right)$, depends on the continuous strain field $\bar{\varepsilon}^{u}$, and the free discrete energy density, $\Psi_{S}([|\mathbf{u}|])$, depends on the jump. These energy densities are respectively given by:

$$
\begin{gathered}
\Psi\left(\overline{\boldsymbol{\varepsilon}}^{u}\right)=\int_{0}^{\bar{\varepsilon}} \sigma(\bar{\varepsilon}) d \bar{\varepsilon} \\
\phi_{S}([|\mathbf{u}|])=\int_{0}^{[|\mathbf{u}| \mid} \mathbf{T}_{S}\left([|\mathbf{u}|]_{n, s}\right) d[|\mathbf{u}|]
\end{gathered}
$$

where the elastic stresses, $\sigma$, are defined by:

$$
\sigma=\mathbf{C}: \bar{\varepsilon}
$$

and $\mathbf{T}_{\mathrm{S}}$ is the traction vector at the discontinuity.

\subsection{Approximation of the displacement and strain fields}

The regular displacement field is approximated by: 


$$
\hat{\mathbf{u}}=\mathbf{N d}
$$

where $\mathbf{N}$ is the standard vector of shape functions of the element

$$
\mathbf{N} \equiv \sum_{i=1}^{i=n} N_{i}^{(e)}
$$

and, $\mathbf{d}$, is the nodal displacement vector. The function, $M_{S}(\mathbf{x})$, is defined in the finite element approximation as:

$$
M_{S}^{e}(\mathbf{x})=H_{S}^{e}(\mathbf{x})-\phi^{e}
$$

where $\phi^{e}$ is constructed by:

$$
\phi^{e}=\sum_{i^{+}=1}^{n_{e}^{+}} N_{i^{+}}
$$

where $N_{i^{+}}$are the shape functions corresponding to the nodes placed on $\Omega^{+}$of the finite element which contains the discontinuity. The displacement is given by

$$
\mathbf{u}=\mathbf{N} \mathbf{d}+\underbrace{M_{S}^{e}}_{\mathbf{N}_{c}}[|\mathbf{u}|]_{x, y}
$$

The continuous strain field is approximated as:

$$
\bar{\varepsilon}=\mathbf{B} \cdot \mathbf{d}-\underbrace{\nabla \phi^{e}}_{\mathbf{B}_{c}} \cdot[|\mathbf{u}|]_{x, y} \quad \forall \mathbf{x} \in \Omega / S
$$

Where $\mathbf{B}$, is the standard strain interpolation matrix, containing the derivatives of the standard shape functions $\partial(\mathbf{N d})=\mathbf{B d}$.

The equilibrium equations corresponding to this formulation are obtained by substituting Eqs. (9) and (10) into the energy functional of Eq. (1), and setting the derivatives with respect to the independent variables ( $\mathbf{d}$ and $[|\mathbf{u}|])$ to zero,

$$
\begin{gathered}
\frac{\partial \Pi}{\partial \mathbf{d}}=0=\int_{\Omega \backslash S} \mathbf{B}^{T} \boldsymbol{\sigma}(\overline{\boldsymbol{\varepsilon}}) d \Omega-\int_{\Omega} \mathbf{N}^{T} \cdot \mathbf{b} d \Omega-\int_{\Gamma_{\sigma}} \mathbf{N}^{T} \cdot \mathbf{t}^{*} d \Gamma \\
\frac{\partial \Pi}{\partial[|\mathbf{u}|]}=0=-\int_{\Omega \backslash S} \mathbf{B}_{c}^{T} \boldsymbol{\sigma}(\overline{\boldsymbol{\varepsilon}}) d \Omega+\int_{\Gamma_{S}} \mathbf{T}_{x, y} d \Gamma
\end{gathered}
$$

In Eqs. (11) and (12), $\boldsymbol{\sigma}(\bar{\varepsilon})$ and $\mathbf{T}_{x, y, z}$ are nonlinear, their respective linearizations with Taylor series give [5]:

$$
\left[\begin{array}{cc}
\int_{\Omega \backslash S} \mathbf{B}^{\mathbf{T}} \cdot \mathbf{C} \cdot \mathbf{B} d \Omega & -\int_{\Omega \backslash S} \mathbf{B}^{\mathbf{T}} \cdot \mathbf{C} \cdot \mathbf{B}_{c} d \Omega \\
-\int_{\Omega \backslash S} \mathbf{B}_{c}^{\mathbf{T}} \cdot \mathbf{C} \cdot \mathbf{B} d \Omega & -\int_{\Omega \backslash S} \mathbf{B}_{c}^{\mathbf{T}} \cdot \mathbf{C} \cdot \mathbf{B}_{c} d \Omega+\int_{\Gamma_{S}} \mathbf{R}^{T} \cdot \mathbf{T} \cdot \mathbf{R} d \Gamma
\end{array}\right]^{(n, 0)}\left\{\begin{array}{c}
\Delta d \\
\Delta[|\mathbf{u}|]_{x, y}
\end{array}\right\}^{(n, 1)}=\left\{\begin{array}{l}
R_{1} \\
R_{2}
\end{array}\right\}^{(n, 0)}
$$

where $\mathbf{R}$ has the direction cosines, $R_{1}$ and $R_{2}$ are defined as:

$$
\begin{gathered}
R_{1}=F_{e x t}^{(n)}-\int_{\Omega \backslash S} \mathbf{B}^{T} \boldsymbol{\sigma}(\overline{\boldsymbol{\varepsilon}}) d \Omega^{(n, 0)} \\
R_{2}=\int_{\Omega \backslash S} \mathbf{B}_{c}^{T} \boldsymbol{\sigma}(\overline{\boldsymbol{\varepsilon}}) d \Omega^{(n, 0)}-\int_{\Gamma_{S}} \mathbf{T}_{x, y} d \Gamma^{(n, 0)}
\end{gathered}
$$

To reduce the size of the system given in Eq. (13), the additional degrees of freedom, $\Delta[|\mathbf{u}|]$, may be condensed. In Eq. (14), $R_{1}$ means the equilibrium between the external and the internal forces in the domain $\Omega \backslash S$, whereas $R_{2}$, in Eq. (15), the equilibrium between the forces in the domain $\Omega \backslash S$ and forces in the discontinuity $\Gamma_{S}$.

Tractions at the discontinuity are:

$$
\mathbf{T}_{x, y, z}=\frac{1}{A_{d}} \int_{\Omega \backslash S} \mathbf{B}_{c}^{T} \sigma d \Omega
$$


which expressed in the local system becomes

$$
\mathbf{T}_{n, s, t}=\frac{1}{A_{d}} \mathbf{R} \int_{\Omega \backslash S} \mathbf{B}_{c}^{T} \boldsymbol{\sigma} d \Omega
$$

The definitions of the traction vector in Eqs. (16) and (17) are dependent on the discontinuity area, $A_{d}$, and the direction cosines to the normal vector $\mathbf{n}$, as shown in Figure 1.
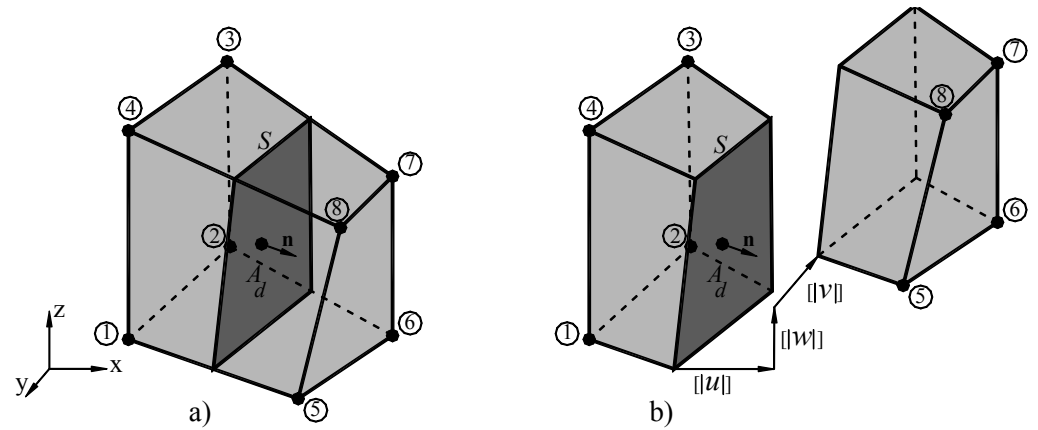

b)

Figure 1: Finite element: a) discontinuity area and b) displacement jumps.

The FEED, given in Eq. (13), were implemented in the finite element analysis program (FEAP), developed by [10]. These FEED capture a discontinuity surface at their geometric centre, which is placed perpendicular to the major principal stress direction. The discontinuity surfaces of the surrounding elements are not aligned and the displacement jump is constant into these FEED. It is important to say that these elements do not have problems of spurious shear deformations and they satisfied the following requirements: (1) equilibrium, traction continuity across the discontinuity interface and (2) kinematics, free relative rigid body motions of the two portions of an element split up by a discontinuity [5].

\section{CONSTITUTIVE MODELS}

\subsection{Concrete}

The concrete and mortar behaviour were modelled with a discrete damage model, which has different threshold values under tension and compression, as shown in Figure 2a. This model is equipped with softening after reaching the ultimate tensile strength, $\mathrm{T}_{u t}$, or the ultimate compressive strength, $\mathrm{T}_{u c}$, shown in Figure $2 \mathrm{~b}$. This model is defined by the following equations: 


$$
\begin{aligned}
& \text { Discrete free energy density } \phi([|\mathbf{u}|], \bar{\alpha})=(1-\omega) \phi_{0}(\bar{\alpha}),\left\{\begin{array}{c}
\phi_{0}([|\mathbf{u}|])=\frac{1}{2}[|\mathbf{u}|] \cdot \mathbf{Q}^{\mathbf{e}} \cdot[|\mathbf{u}|] \\
\mathbf{Q}^{\mathbf{e}}=\mathbf{n} \cdot \mathbf{C} \cdot \mathbf{n}
\end{array}\right. \\
& \text { Constitutive equation } \quad \mathbf{T}=\frac{\partial \phi([|\mathbf{u}|], \bar{\alpha})}{\partial[|\mathbf{u}|]}=(1-\omega) \mathbf{Q}^{\mathbf{e}} \cdot[|\mathbf{u}|] \\
& \text { Damage variable } \\
& \omega=1-\frac{\bar{q}(\bar{\alpha})}{\bar{\alpha}} ; \omega \in[-\infty, 1] \\
& \text { Evolution law } \\
& \dot{\bar{\alpha}}=\bar{\lambda}=\frac{\partial}{\partial t}(\bar{\alpha}), \quad \bar{\alpha} \in[0, \infty] \\
& \text { Damage criterion } \\
& f(\mathbf{T}, \bar{q})=\tau_{\mathbf{T}}-\bar{q} ; \quad \tau_{\mathbf{T}}=\|\mathbf{T}\|_{\mathbf{Q}^{\mathbf{e}^{-1}}}=\sqrt{\mathbf{T} \cdot \mathbf{Q}^{\mathbf{e}^{-1}} \cdot \mathbf{T}} \\
& \dot{\bar{q}}(\bar{\alpha})=\overline{\bar{H}} \dot{\bar{\alpha}} ; \quad \bar{H}=\bar{q}^{\prime}(\bar{\alpha}) \leq 0 \\
& \text { Hardening rule } \\
& f \leq 0 ; \quad \bar{\lambda} \geq 0 ; \quad \bar{\lambda} f=0 ; \quad \bar{\lambda} \dot{f}=0 \text { (consistency) }
\end{aligned}
$$

where $\phi$ is the discrete free energy density, $\mathbf{T}$ is the traction vector. The damage variable $\omega$

is defined in terms of the hardening/softening variable $\dot{\bar{q}}$, which is dependent on the hardening/softening parameter. The damage multiplier $\bar{\lambda}$ determines the loading-unloading conditions, the function $f(\mathbf{T}, \bar{q})$, bounds the elastic domain defining the damage surface in the tractions space. The tangent constitutive equation, in terms of rates from the model in Eq. (18), is:

$$
\dot{\mathbf{T}}=\mathbf{C}_{d}^{T} \cdot[|\dot{\mathbf{u}}|]
$$

where $\mathbf{C}_{d}^{T}$ is the tangent constitutive operator, relating the traction and the displacement jump of the nonlinear loading interval, which is defined by

$$
\mathbf{C}_{d}^{T}=(1-\omega) \mathbf{Q}^{\mathbf{e}}-\frac{\bar{q}-\bar{H} \bar{\alpha}}{\bar{\alpha}^{3}}\left(\mathbf{Q}^{\mathbf{e}} \cdot[|\mathbf{u}|] \otimes[|\mathbf{u}|] \cdot \mathbf{Q}^{\mathbf{e}}\right)
$$

and for the elastic loading and unloading interval $(\dot{d}=0$ and $\dot{\omega}=0)$ :

$$
\mathbf{C}_{d}^{T}=(1-\omega) \mathbf{Q}^{\mathbf{e}}
$$

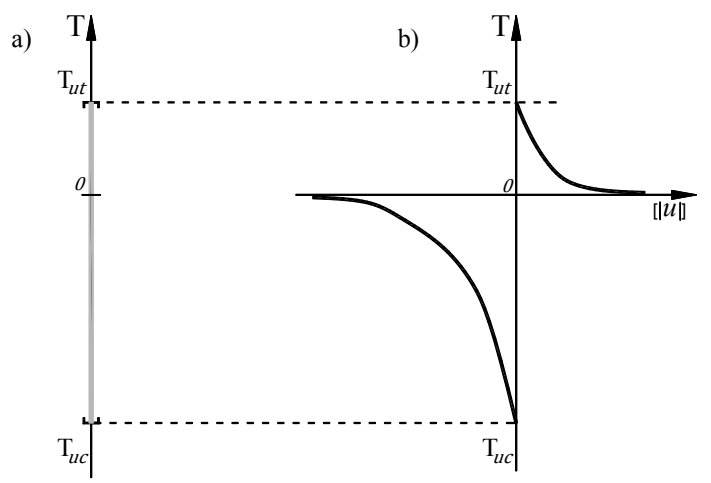

Figure 2: 1D discrete damage model with softening: a) elastic space and b) traction-displacement jump curve. 


\section{NUMERICAL MODELS}

\subsection{Description of the wallets}

To define an indirect shear strength $v_{m}^{*}$ for design and the shear modulus $G_{m}^{*}$ under diagonal tension, small square masonry subassemblies, called wallets, can be tested under axial compression according to NTCM-2004 [7], as shown in Figure 3. This test is commonly performed using an universal press machine [11].

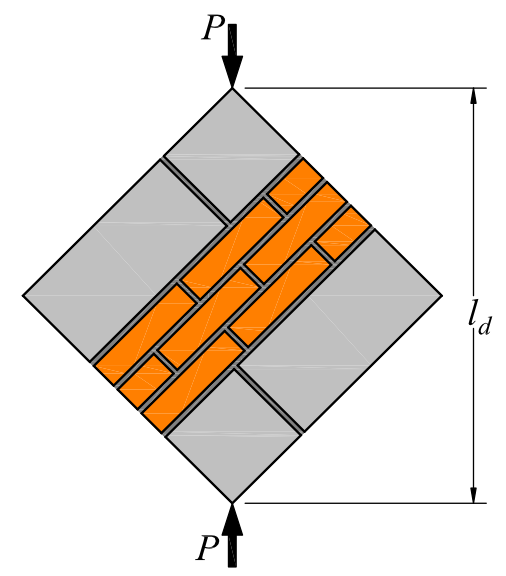

Figure 3: Axial compression test of NTCM-2004.

The shear stress $\tau$ was computed by the following relationship:

$$
\tau=\frac{P}{l_{d} t}
$$

where $\mathrm{P}$ is the vertical load, $l_{d}$ is the diagonal length, measured from the bottom to the top of the wallet and $t$ is the thickness of the wallet, as shown in Figure 3.

The shear strain $\gamma$ was computed as follows:

$$
\begin{gathered}
\gamma=\left|\varepsilon_{c}\right|+\left|\varepsilon_{t}\right| \\
\varepsilon_{c}=\frac{\delta_{c}}{l o_{c}} ; \quad \varepsilon_{c}=\frac{\delta_{c}}{l o_{t}}
\end{gathered}
$$

where $\varepsilon_{c}$ is the average of the compression diagonal strain, $\varepsilon_{t}$ is the average of the tension diagonal strain, $\delta_{\mathrm{c}}$ is the shortening which is measured along the length of the calibrated compression diagonal, $\delta_{\mathrm{t}}$ is the shortening which is measured along the length of the calibrated tension diagonal, $l o_{c}$ is the calibrated length between transducers of the compression diagonal and $l o_{t}$ is the calibrated length between transducers of the tension diagonal. Both lengths, $l o_{c}$ and $l o t$, were measured before the test as shown in Figure 4. 

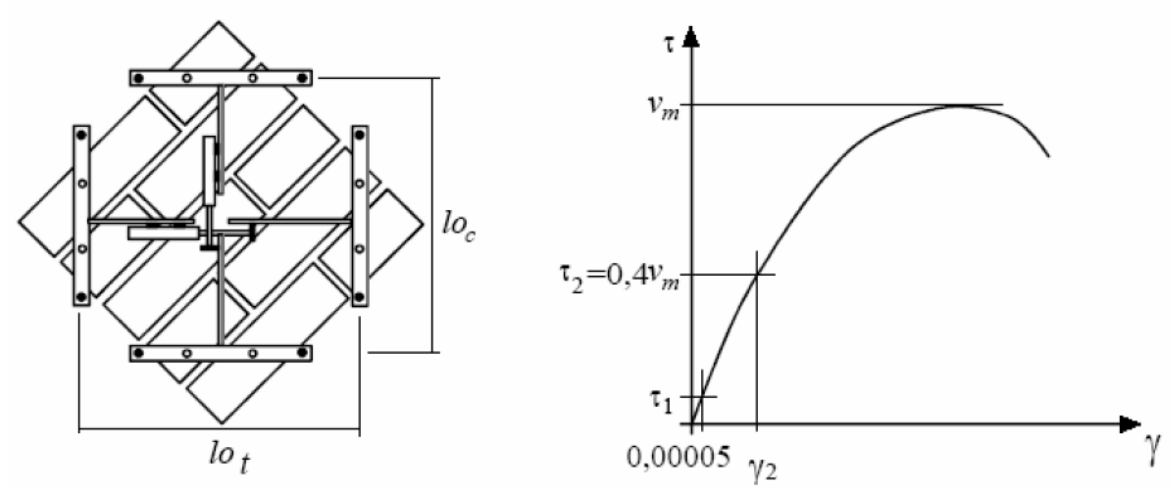

Figure 4: Computation of the shear stress (adapted from [9]).

In this paper, only two arrangements shown in Figure 5 were modelled, with mortar type I, although others two arrangement with mortar type II were also tested by [11]. The mechanical properties of the blocks are: Young's modulus $E=1.1 \mathrm{GPa}$, Poisson ratio $\mathrm{v}=0.2$, ultimate tensile strength $\sigma_{\mathrm{tu}}=0.84 \mathrm{MPa}$, ultimate compressive strength $\sigma_{\mathrm{uc}}=4.26 \mathrm{MPa}$ and fracture energy density $\mathrm{G}_{\mathrm{f}}=15.2 \mathrm{~N} / \mathrm{m}$. The mechanical properties of the fired clay bricks are: Young's modulus $\mathrm{E}=2.0 \mathrm{GPa}$, Poisson ratio $\mathrm{v}=0.1$, ultimate tensile strength $\sigma_{\mathrm{tu}}=2.02 \mathrm{MPa}$, ultimate compressive strength $\sigma_{\mathrm{uc}}=10.1 \mathrm{MPa}$ and fracture energy density $\mathrm{G}_{\mathrm{f}}=13.6 \mathrm{~N} / \mathrm{m}$. The mechanical properties of the mortar are: Young's modulus $\mathrm{E}=2.0 \mathrm{GPa}$, Poisson ratio $\mathrm{v}=0.2$, ultimate tensile strength $\sigma_{\mathrm{tu}}=4.82 \mathrm{MPa}$, ultimate compressive strength $\sigma_{\mathrm{uc}}=24.1 \mathrm{MPa}$ and fracture energy density $\mathrm{G}_{\mathrm{f}}=7.5 \mathrm{~N} / \mathrm{m}$. The fracture energy density and Young's modulus were computed by [8].
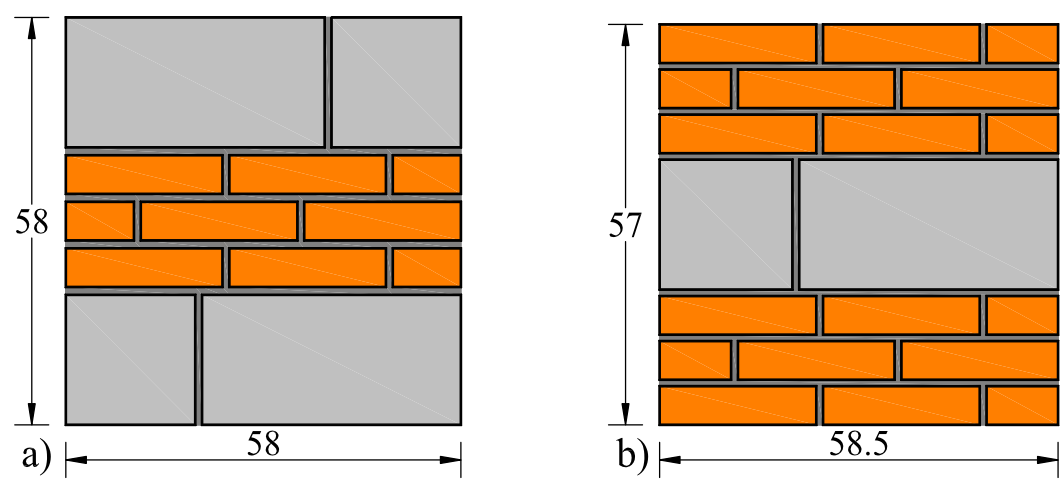

Figure 5: Small wallet arrangements: a) 1 and b) 2 (adapted from [11]).

\subsection{Numerical test}

The wallet of the arrangement 1 was discretized with quadrilateral elements, 2052 elements for blocks, 840 elements for bricks and 298 for joints as shown in Figure 6a; whereas the wallet of the arrangement 2 was discretized with 1008 elements for blocks, 1650 elements for bricks and 298 for joints as shown in Figure 6b. Homogenization techniques were not use, so the mechanical properties of the blocks, bricks and joints were assigned to the corresponding discretized elements. 
a)

b)

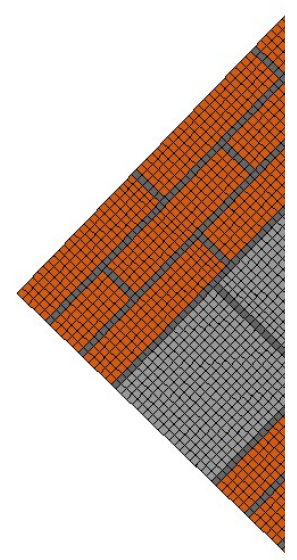

Figure 6: Discretization of the wallets, arrangement: a) 1 and b) 2 .

The average shear stress $v s$ shear strain curves are shown in Figure 7. The computed curve with FEED shows numerical results, which are congruent at the ascending branch with the experimental curve reported by [4]. It is observed that both curves are similar at the beginning; however, the curves computed with FEED are stiffer that the experimental curves. This difference may be attributed to the fact that the sliding between mortar and blocks or bricks were not considered.

a)

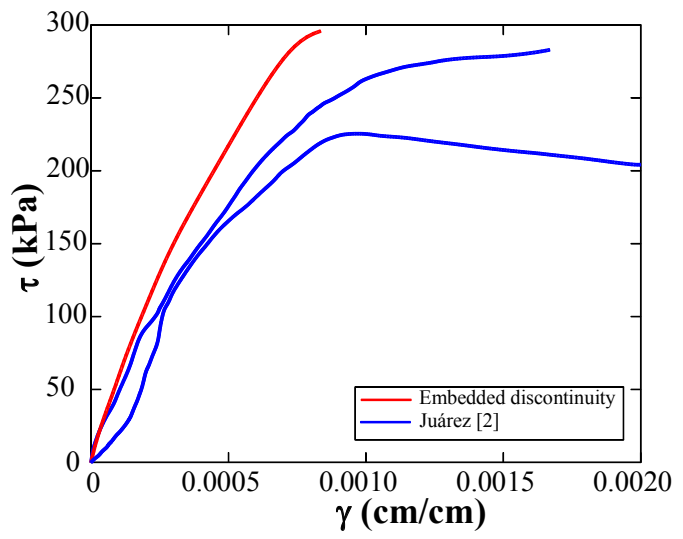

Figure 7: Shear stress $v s$ shear strain for arrangement a) 1 and b) 2

In both arrangements, cracking initiated at both corners, on the top and on the bottom, growing to the centre. Discontinuities along bed joints occurred in arrangement 1 as shown in Figure 8a, whereas discontinuities along the diagonal occurred in the arrangement 2 as shown in Figure 8. These crack patterns at both arrangements were congruent with the experimental results reported by [4] and [11]. 
a)

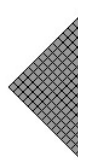

b)

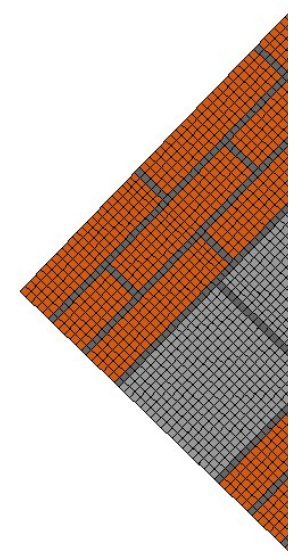

Figure 8: Cracking patterns for arrangement: a) 1 and b) 2

\section{CONCLUSIONS}

- The FEED with a discrete damage model were used for modelling wallets under compression load, involving their crack pattern and the shear stress-shear strain capacity curve. The discrete damage model considers a constitutive behaviour equipped with softening for the blocks, bricks and joints elements limited for a damage surface.

- The coupling of bricks or block with the mortar yield a shear stress- shear strain curve stiffer than the experimental curves, because sliding is neglected.

- In the wallet 1 , discontinuities occurs along bed joints, whereas in the wallet 2 , discontinuities occur along the diagonal, which are congruent with the experimental results.

- The ultimate shear stresses computed with FEED were greater than experimental values. These effect is attributed to absence of the sliding in the brick-mortar interface. Forcoming work will include the sliding of brick-mortar interfaces.

\section{Acknowledgements}

The first author acknowledges the financial support given by CONACYT under the agreement number I010/176/2012, in the context of the research project: "Analysis and design of concrete slabs". Both authors acknowledge the sponsorship by the project 269222 entitled Analysis and Design of Earthquake Resistant Structures (ADERS) and the support given by the Universidad Autónoma Metropolitana.

\section{REFERENCES}

[1] J. Alfaiate, A. Simone, L.J. Sluys, Non-homogeneous displacement jumps in strong embedded discontinuities. International Journal of Solids and Structures, 40(21), 57995817, 2003. 
[2] P.G. Asteris, D.M. Cotsovos, C.Z. Chrysostomou, A. Mohebkhah, G.K. Al-Chaar, Mathematical micromodeling of infilled frames: state-of-the-art. Engineering Structures, 56, 1905-1921, 2013.

[3] P.G. Asteris, M.P. Chronopoulos, C.Z. Chrysostomou, H. Varum, V. Plevris, N. Kyriakides, V. Silva, Seismic vulnerability assessment of historical masonry structural systems. Engineering Structures, 62-63,118-134, 2014

[4] A. Juárez, Mecanismos de resistencia y de deformación de muros de mampostería combinada y confinada. M. Sc. Thesis. División de Ciencias Básicas e Ingeniería, Universidad Autónoma Metropolitana Azcapotzalco, 2008. (In Spanish).

[5] G. Juárez-Luna, G.A. Ayala, Improvement of some features of finite elements with embedded discontinuities. Engineering Fracture Mechanics, 118, 31-48, 2014.

[6] H.R. Lotfi, P. Shing, Embedded representation of fracture in concrete with mixed finite elements. International Journal for Numerical Methods in Engineering, 38(8), 1307$1325,1995$.

[7] NTCM-2004, Normas Técnicas Complementarias para Diseño y Construcción de Estructuras de Mampostería. Gaceta Oficial del Distrito Federal, October, 2004. (in Spanish)

[8] R. Illampas, Personal communication, 2015.

[9] V. Salinas, Comportamiento ante cargas laterales de muros de mampostería combinada unidos con morteros de autoconstrucción. M. Sc. Thesis. División de Ciencias Básicas e Ingeniería. Universidad Autónoma Metropolitana Azcapotzalco, 2008. (In Spanish).

[10] L.R. Taylor, A finite element analysis program (FEAP) v8.2. Department of Civil and Environmental Engineering, University of California at Berkeley, Berkeley, CA 2008.

[11] A. Tena-Colunga, A. Juárez-Ángeles, V.M. Salinas-Vallejo, Cyclic behaviour of combined and confined masonry walls. Engineering Structures, 31(1), 240-259, 2009.

[12] G.N. Wells, L.J.Sluys, A new method for modelling cohesive cracks using finite elements. International Journal for Numerical Methods in Engineering, 50(12), 2667-2682, 2001 . 